\title{
Proceeding
}

Supplementary Issue: Summer Conferences of Sports Science. Costa Blanca Sports Science Events, 25-26 September 2020. Alicante, Spain.

\section{Immune system and physical activity}

\author{
VINCENZO CRISTIAN FRANCAVILLA 14 , RITA POLITO², GIUSEPPE MESSINA ${ }^{3}$, MARIA CHIARA \\ PARISI ${ }^{1}$, OMAR GAETANO MARIA MINGRINO ${ }^{1}$, ANGELO CAMPANOZZI ${ }^{4}$, AURORA DANIELE ${ }^{5}$, \\ ANTONIETTA MESSINA ${ }^{6}$, VINCENZO MONDA ${ }^{5}$, ANNA VALENZANO ${ }^{7}$ \\ ${ }^{1}$ School of Engineering, Architecture, and Motor Sciences, Kore University of Enna, Enna, Italy \\ 2Department of Public Health, University of Naples "Federico II", Naples, Italy \\ ${ }^{3}$ Posture and Biomechanics Laboratory, Department of Psychology, Educational Science and Human Movement, \\ University of Palermo, Palermo, Italy \\ ${ }^{4}$ Department of Medical and Surgical Sciences, University of Foggia, Italy \\ ${ }^{5}$ Department of Biological and Pharmaceutical Environmental Sciences and Technologies, Luigi Vanvitelli University \\ of Campania, Naples, Italy \\ ${ }^{6}$ Department of Experimental Medicine, Section of Human Physiology and Unit of Dietetic and Sport Medicine, Luigi \\ Vanvitelli University of Campania, Naples, Italy \\ ${ }^{7}$ Department of Clinical and Experimental Medicine, University of Foggia, Italy
}

\begin{abstract}
Regular physical activity and sport are able to modify the diffused neuro-immuno-endocrine-endothelial system. This emerges from careful scientific research that finds deep interconnections between the psyche, the nervous system, endocrine organs and the immune response. There is an interconnection between the psychoendocrine-immunological system and respiratory infections in athletes. The appearance of leucocytosis during intense physical activity can explain to us how strenuous exercise is responsible for a temporary depression of the immune system; indeed, the physical activity reduces the proliferative activity and function of T-lymphocytes and Natural Killer cells. This is associated with the influence of multiple factors such as age and gender. The respiratory mechanics during strenuous physical activity favours the dehydration of the mucous membranes, the modification of the mucociliary clearance and therefore the predisposition to infections, until the appearance of more complicated diseases such as neoplasms, as reported by data literature. In conclusion, regular and moderate exercise improves the immune system, reduces environmental and psychological stress, decreases the demand and administration of chemotherapy and immunosuppressants.
\end{abstract}

Keywords: Immunity; Exercise; Physical activity; Neoplasm.

\section{Cite this article as:}

Francavilla, V.C., Polito, R., Messina, G., Parisi, M.C., Mingrino, O.G.M., Campanozzi, A., Daniele, A., Messina, A., Monda, V., \& Valenzano, A. (2020). Immune system and physical activity. Journal of Human Sport and Exercise, 15(4proc), S1534-S1543. doi:https://doi.org/10.14198/ihse.2020.15.Proc4.49

Corresponding author. School of Engineering, Architecture, and Motor Sciences, Kore University of Enna, Enna, Italy.

E-mail: vincenzo.francavilla@unikore.it

Abstract submitted to: Spring Conferences of Sports Science. Costa Blanca Sports Science Events, 19-20 June 2020. Alicante, Spain.

JOURNAL OF HUMAN SPORT \& EXERCISE ISSN 1988-5202

(c) Faculty of Education. University of Alicante

doi:10.14198/jhse.2020.15.Proc4.49

S1534 | 2020| Proc4 | VOLUME 15

C 2020 University of Alicante 


\section{INTRODUCTION}

As defined by the World Health Organization (WHO), health is a state of complete physical, mental, and social well-being and not merely the absence of disease and disability. Health promotion and protection number among the fundamental rights of citizens, and as such, they come under the responsibility of the State. Accordingly, the national health service is mandated to ensure the provision of health information and education, with a view to raise awareness of healthy lifestyles among citizens and public authorities. Sports is an integral part of a society, its culture, traditions, and progress. Technological innovations in sports have significantly extended the opportunities for social and active inclusion of the handicapped. Sports and physical activity hold a recognized role in promoting lifestyles that maintain health as an overall condition of well-being and not merely "the absence of dis- ease". There is ample evidence that physical exercise protects against cardiovascular, bone and joint, and metabolic disorders and that it is an effective means in the prevention and treatment of certain types of cancer (Gleesons et al., 2000). A theme of great scientific importance, that for a while arouses the interest of physiologists, immunologists and sports medicine specialists, is the alleged correlation among immune system and sport activity. The research developed on the matter, besides scanty and effected on a limited number of athletes, have often produced incomplete or discordant results, but nevertheless, they have underlined some effects induced by the physical exercise on the immune function (Pedersens et al., 1997). In relationship to the deep interconnections among psyche, nervous system, endocrine organs and immune function, rather than of immune system is more correct to speak of system "psychoneuroendocrine-immunological" (PNEI): the answer to stress events of different nature involves, in fact, the organism in its totality, both to psychic level and of the central nervous system, and to peripheral level (Peterses et al., 1997). The search is directed on two seams: the first one concerns the effects of physical effort on the immune function, on the activity of the different components of the immune system and the interconnections among the latter and the neuroendocrine system; the second aim to define the supposed and discussed predisposition of the athletes to infections, particularly those of the upper respiratory tract, which may be responsible for the onset of more serious diseases such as cancer (Francavilla et al., 2018). For these reasons, the aim of this review is to analyse the interconnection between immune system and physical activity, focusing on their role also in development and treatment of cancer disease.

\section{PHYSICAL EXERCISE AND IMMUNE SYSTEM}

Upon a careful revision of the scientific studies carried out, it is possible to extrapolate some certain data on the acute and chronic effects of the physical exercise on the different components of the immune system and on the risk of upper respiratory tract infections (URTI). A critical revaluation of the data in scientific literature is necessary to work out an adequate prevention and timely treatment of the viral infections in the athletes (Francavilla et al., 2018; 2017; 2013; Gleesons et al., 2000).

The susceptibility to the respiratory infections is influenced both from the patterns of respiratory flow, the presence of viruses and carcinogens in suspension in the air inhaled during the physical exercise and from the mechanical barriers, represented by the respiratory epithelium and by the mucus. The tracheal epithelium performs a continuous movement of the mucus and the particles trapped in it towards the outside, favoured by cough and sneeze, and finalized to the elimination of the particles with expectoration.

The respiration through mouth, used often during physical effort, causes a progressive cooling and desiccation of the respiratory mucosa, with deceleration of the ciliary movement, increase of the mucus 
viscosity and reduction of the microorganism clearance. Also, the mucosa B-lymphocyte function and the local antibody secretion can be impaired (Table 1) (Polito et al., 2020)

Table 1. Defended physics and biological against the viral infections and the neoplastic cells.

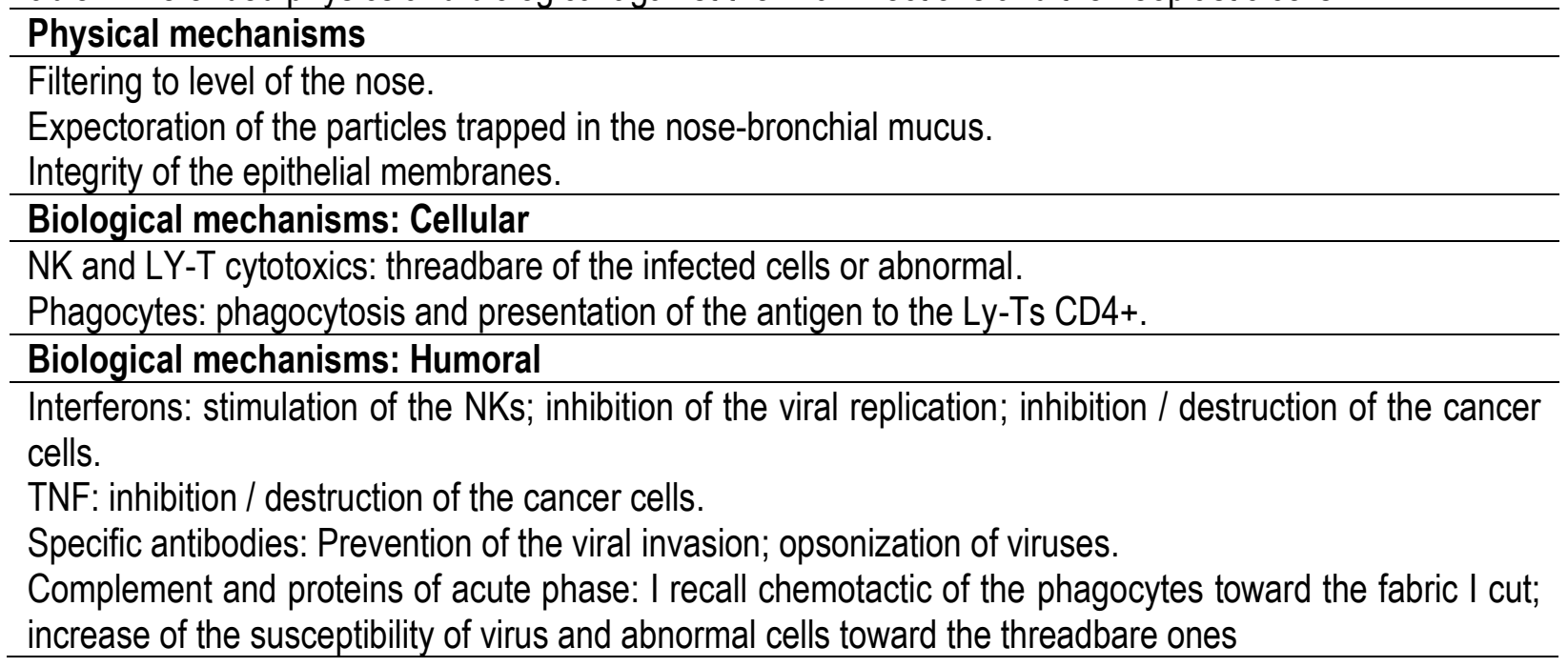

There is evidence that virus susceptibility increases after a marathon run, but doesn't suffer any modification after a 20-30 km run, even if the impact on the physical barriers is equal: it seems therefore unlikely that the damage on the mechanical defences accounts by itself for the increased susceptibility to respiratory infections in subjects that train heavily. Seems therefore necessary to revalue the role of the principal biological mechanism of defence against infections, represented by the immune system, which is activated more slowly because characterized by a sequence of events, from phagocytosis of the infectant microorganism, to intracellular killing, to the elaboration of the surface proteins for antigen presentation to the Ly-Ts in association to MHC proteins and secretion of cytokines as IL-1 and IFN-alpha.

It is shown that specific cytotoxic lymphocytes proliferation reaches its maximum in about 7 days after the initial stimulus (Francavilla et al., 2016; 2015; 2017). During physical exercise, the leucocytosis appears in relationship to the intensity and the duration of effort, probably by effect of the catecholamines that stimulate the separation of the Neutrophils from endothelium. The circulating neutrophils count often increases notably during and sometimes after exercise, nevertheless without modification of resistance towards secondary bacterial infections that, sometimes, might complicate and prolong the viral respiratory infections, since phagocytic activity can simultaneously decrease.

It has been observed in isolated neutrophils that intensive training can alter the oxidative potential associated to killing, but the susceptibility of the athlete towards respiratory infections doesn't necessarily seem affected (Valenzano et al., 2019; Francavilla 2007). At the end of physical effort, the neutrophilia re-enters in the norm, but reappears after some time, because of the release in circle of the mature neutrophils induced by cortisol. During prolonged exercise there is a massive influx of polymorphonuclear leukocytes to the mucous membranes of the respiratory tract, reproducing a picture that can be superimposed on that of inflammation and considered harmful to the defensive barrier of the mucosa (Di Maio et al., 2020; Polito et al, 2020; Francavilla et al., 2018; 2016). According to some authors, the strenuous exercise would be responsible of a reduction in the rate of serum complement, $C$ reactive protein, power of adhesion and phagocytic activity of the neutrophils, pathognomonic signs of depression, even though temporary, of the immune system. The macrophages count increases with exercise but returns to normal values within a few hours following the end 
of physical activity. The moderate exercise stimulates the activity cytostatics of the macrophages, increasing TNF-a production; nevertheless, it is observed, subsequently to a brave effort and prolonged, kind if repeated for more consecutive days, a meaningful compromising of the activity antimicrobic of the alveolar macrophages, with reduction of the phagocytic activity also quite a lot days after the run. The macrophages activity is reduced subsequently to a very heavy exercise, but also from the prostaglandin PGE2, both that this is produced by a muscular microtrauma that from a tumour. The acute exercise would be also responsible for a monocytosis, and particularly of an immediate increase, intensity-dependent, of the number and of the cytolytic activity of the Natural Killer (NK) currencies, responded also mimed by physiological doses of catecholamine administered to rest (Corbi et al., 2019; Monda et al., 2019). Nevertheless, such increase, that should strengthen the resistance to the infections and the cells tumoral, would only persist during the exercise, brief period excessively because it has clinical effects. Immediately after a vigorous training the cells NKs considerably reduce, even if in the turn of few times they re-enter in the normal values; if the I strive it extends for some times, the cells NKs gradually re-enter within the basal values (Moscatelli et al., 2016).

It counts her basal of the NKs it would increase, instead, following repeated sessions of moderate physical exercise; this could explain because the immune defences are strengthened at times in the people that practice in constant way a physical activity of moderate intensity. The IL-1 possesses a cytotoxic action, stimulating the Ly-Ts to produce superior quantity of IL-1 and IL-2, with the effect to strengthen the cytotoxicity of the NK cells. The physical exercise stimulates the production of IL-1 and the training increases the basal levels of it (Gleesons et al., 2000; Pedersens et al., 1997).

The IL-2, released by the CD4 + T lymphocytes, represents for a long time the principal defence term against the viral infections and the neoplastic cells, in how much it stimulates the proliferation and the function of the Ly-Ts and the cells NK (Peterses et al., 1997).

Intense physical activity reduces this proliferative response, even for a few times after the end of the exercise, contributing to the window of opportunity for viruses and neoplastic cells. On the other hand, moderate training would be able to limit this proliferative depression induced by a single episode of intense exercise. In addition, the interferons (IFN) increase the lytic activity of macrophages and NK cells (Gleesons et al., 2000; Pedersens et al., 1997).

Moderate training can increase the production of IFN, but after a few weeks of strenuous training the production of the IFN range would be unchanged.

The tumour necrosis factor (TNF), produced by monocyte possesses a cytotoxic action, stimulating the activity of macrophages, $T$ and $B$ lymphocytes, and contributes significantly to the muscular deterioration. The TNF- $\beta$, released by the activated Ly-Ts, it is endowed with action both cytostatic and cytotoxic against the cells tumoral. The acute exercise increases the production of TNF, but the effect of the training is still unknown. After a courageous effort there is also an increase in the serum concentration of some Interleukins, in particular IL-6 and IL-8. The latter inhibits the migration of neutrophils into the extravascular centre and therefore into the foci of infection. Furthermore, the $B$ lymphocyte, a reduction in circulating levels is highlighted during and after intense training, at the same time as the reduction of CD4 + T lymphocyte. Whatever diminution of the cells CD4 + limits the production of the cytokines that they activate the cells NK and the Ly-Ts and they stimulate the proliferation and maturation of the Ly-Bs: the maintenance of a relationship superior CD4 + / CD8 + to 1.5 e's therefore important for the immune defences. Excessive training and heavy exercise can cause this ratio to decrease, but more information is needed to understand the importance of those changes. Plasma immunoglobulin levels would generally rise after submaximal 
exercise but would subsequently decrease with major effort. In particular, the moderate exercise would not modify the concentration of the serum IgGs and would increase the salivary lgAs, while the very intense exercise would reduce the concentrations of $\lg A$ both in the saliva and in the tampon (washing) nasal. A small diminution of the serum lgGs has also been observed in athletes of elite during the period of maximum training. The diminution of the mucosal IgAs could have an important influence on the immune defences, from the moment that the secretive IgAs inhibit the taking root of the viruses to the respiratory epithelium and the following intracellular replication. Many studies have documented a correlation among diminution of the salivary IgAs and increase of the prevalence of infections of the tall respiratory streets (Patti et al., 2017). It is conceivable that a moderate physical activity can be regulate the cytokines levels and to maintain trained the immune system, but excessive programs of training and brave exercises can exhaust the defensive abilities of the organism favouring the to establish him of immunodeficiencies (Gleesons et al., 2000).

\section{PHYSICAL EXERCISE AND SUSCEPTIBILITY TO THE INFECTIONS}

The answer of the immune system to the physical exercise is notably variable and it is influenced by many factors, such as age, sex, the intensity and the duration of the effort. Some physiologist of the sport have postulated a relationship to " $J$ " between physical activity and susceptibility to the viral infections, according to which a moderate physical activity and to regulate would be able to strengthen the answer immune cellular and humoral, reducing the incidence of acute infectious pathologies of the tall respiratory streets and the severity and the duration of the symptomatology (Mazzeo et al., 2013). An intense physical effort as a program of excessive and inadequate training, would have been suppressing contrarily, the immunity for some times until to a week or more, creating a brief period of vulnerability during which the risk of infections of the superior aerial streets increases. Nevertheless, such transitory alterations could be you also link to other accidental causes or in partnership circumstances to the exercise (Moscatelli et al., 2015;2016). Nieman, one of the most authoritative researchers of the subject, have noticed that a regular physical exercise, practiced by a champion of 25-45 year-old women, for 45 minutes, five times the week, to an intensity equal to the $60 \%$ of the aerobic ability, it reduces the duration of the respiratory troubles (odynophagia, syndrome influence her, cough, rhinorrhoea), but it doesn't modify the incidence of the infections of the superior aerial streets (Nieman et al., 1993). The benefits of the regular physical activity are more easily evidenced in the elderly subjects, probably in relationship to a condition of characteristic base of the third age and consistent in a function immune depressed and a lower part level of physical activity.

Studies effected on athletes of high-level, mainly cross-country skiers, rowers, fighters, swimmers, have shown a more elevated incidence of infectious respiratory pathologies in comparison to the sedentary or practicing population a moderate physical activity. The participants to a marathon would have a probability 5 times superior to develop infectious episodes in comparison to athletes equally trained, but that they don't participate in the event. It has not been entirely still clarified if such changes must be attribute to a damaged immunity, in how much few studies have gotten the clinical confirmation of the infection or looked for a depressed immune function (Messina et al., 2018; Messina et al., 2016).

In the etiopathogenesis would also intervene some factors concausal, separate in environmental, what the inhalation of cold air, that behaves a reduction of the temperature to level of the mucous ones of the tall respiratory, and individual streets as pre-existing alterations of the mucous, distance in km crossed to week, to live alone, to have a low body mass index. Warm or I cool extreme, tall or low atmospheric pressures, inhalation of polluted air, deprivation of sleep, changes of fused schedule and strengths exposure gravitational tall or low, they are all factors that can negatively influence the immune function. The environmental stresses can induce besides some answers neuroendocrine as the secretion of 
catecholamines in answer to the exposure to the cold or the secretion of cortisol, IL-1 and IL-6 in association to an increase of the central temperature. It doesn't finally need to underestimate the role of the psychological stress, that manages his effects on the immune function and that it acts in cumulative way with the physical exercise, it is also inducing a secretion of epinephrine, norepinephrine, cortisol and hormone of the growth. The athletes that cross more than $92 \mathrm{~km}$ the week would have the double one some probabilities to develop a respiratory infection in comparison to those people that cross 32 less than of it. Some investigations epidemiological underline that $76 \%$ around of the athletes it is considered less vulnerability to the colds in comparison to the sedentary people, and only $1.5 \%$ retain more vulnerability. Nevertheless, $16 \%$ of these athletes he/she thinks about becoming more susceptible to the infections in coincidence with the period of maximum intensity of training or the competitive appointments.

Surely intense physical activity is primarily in partnership to a respiration with the mouth, that favours the dehydration of the mucous ones, it alters the clearance mucociliare and it predisposes to the onset of infections of the superior aerial streets. The agents responsible etiological are, in the greatest part of the cases, virus as the rhinoviruses and the influenza viruses, even if the incidence of the bacterial infections of the tall respiratory streets in the athletes would be taller in comparison to the general population. It is shown that periods of heavier training are in partnership to reduced concentrations of salivary lgA, nevertheless, some athletes (skiing leading, gymnasts, swimmers and fighters) they develop infections of the superior aerial streets with the same frequency of the sedentary people. Presumably susceptibility depends on the type and from the quantity of training: the data epidemiological underline that the moderate physical activity $\mathrm{e}^{\prime}$ associated to an optimal immune function against the infections, while the heavy exercise would condition an ineffective answer, but don't allow to show a clear relationship dose-answered to "J" between volume of developed exercise and function immunitaria yet. A similar scheme e' is also proposed for the neoplasms, but with limited data even more. In conclusion, it can be stated that a single and intense effort increases the risk of respiratory tract infections, while moderate and continuous training reduces the incidence of symptoms attributable to an alteration in the upper airways.

\section{EXERCISE, IMMUNITY AND TUMORS}

Among the manifold benefits associated to the practice of a regular physical exercise, The effects on tumours, in particular of the breast and colon, have been studied in recent times. The results of some randomization studies show a positive correlation among practice to regulate of a physical exercise and reduction of the risk of tumour of the breast: women that develop a sporting activity from 1 to 3 weekly hours would have a $30 \%$ risk reduction, and, increasing the quantity of exercise over the 4 weekly hours, such value percentage can reach $55 \%$. A correlation would likewise, exist among the years of sedentary working activity and the cumulative risk of crab of the colon. For other tumours as those of the uterus, of the cervix, of the prostate and of the bellows, it seems that an inverse correlation exists between incidence and regular practice of physical exercise. The interpretation of these epidemiological data must be based on a multifactorial analysis, due to the many and, unfortunately still partly unknown, etiopathogenetic mechanisms involved in carcinogenesis. Regarding breast cancer, statistically given the existence of a significant correlation with the increase in body fat and obesity, it can be hypothesized that the benefits of regular exercise are more closely linked to better control of body weight. The intensity of the exercise causes an increase in gastrointestinal symptoms such as cramps, diarrhoea, bloating, nausea and bleeding. These problems have been associated with reduced intestinal permeability and reduced intestinal barrier function. The increased gastrointestinal permeability, the so-called intestinal permeability, can lead to endotoxemia, with a consequent increase in susceptibility to infections and autoimmune diseases, caused by the entry into the circulation of pathogens and toxins. The key components that determine intestinal barrier function and gastrointestinal permeability 
are cellular junctions or (tight junctions), protein structures located in the paracellular channels between the epithelial cells and the intestinal wall. The structural and functional integrity of these junctions depends on sophisticated interactions between substances produced by intestinal residents, on the metabolism of intestinal epithelial cells and on the activities of lymphoid tissue associated with mucous membranes. There is evidence that the administration of probiotics positively affects the integrity of the intestinal barrier in acute conditions. Modifications of the intestinal barrier - secondary to "physiological" hypo perfusion, especially in the case of prolonged physical stress, can be responsible for alterations both in the absorption-use of nutrients, and in the modification of immune reactivity and systemic inflammatory state, with effects also on other organs / systems, especially respiratory and urinary systems, with alteration of the function of the latter and decrease in resistance to bacterial and viral infections (Gleesons et al., 2000; Polito et al., 2020). The evidence of a relationship between physical exercise and a decrease in colon cancer, and in general of the gastrointestinal system, seems to depend on a stimulation of the metabolism, gastrointestinal transit, digestive processes and urinary excretion, with faster and better elimination of carcinogenic substances and toxic metabolites introduced with food. In recent years it has been hypothesized that regular physical exercise would reduce the risk of tumours through an action of modulation and support of the immune system, in relation to the role that the latter plays in the shutdown and prevention of the carcinogenesis process (Polito et al., 2020). The hypothesis is confirmed by the fact that patients on immunosuppressive therapy have a high incidence of some types of crab, which form consequent tumours to previous infections following an impairment of the immune system, and that AIDS is related to an incidence higher than neoplasms. An explanation of the lower probability of some cancers among more physically active individuals and regularly apprentices of a sport could be more related to the presence of elevated levels of NK cells, which have been hypothesized to play a first-line defence role against some cancers. An efficient immune system destroys the abnormal cells, initiated in the process of neoplastic transformation, in order to maintain the homeostasis of the organism.

\section{CONCLUSIONS}

Regular and moderate exercise improves the function of the immune system and induces an increase in the number of circulating NK cells. Intense physical exertion can temporarily reduce resistance to viral infections, even if the dose-response correlation at " $\mathrm{J}$ " between exercise and immune function has not yet been confirmed: other factors would intervene, such as nutritional, hygiene, environmental and pharmacological strategies. It seems that professional athletes have a more efficient and more ready immune function than sedentary ones. However, those preparing for international competitions or participating in strenuous races such as marathons should consider taking appropriate measures to minimize the immunosuppression related to intense exercise; such precautions should not be necessary for amateur athletes.

From the study undertaken on the complex relationships between physical exercise and immune systematization, some behavioural guidelines can be extrapolated, aimed at the prevention and treatment of infectious episodes:

1. Threshold exposure to sources of contagion and avoid intimate contact with people who show signs and symptoms of a respiratory infection.

2. Monitor training and adapt workload to physical and nutritional status of athletes.

3. To control the diet, especially the intake of sufficient carbohydrates, proteins containing a balanced amount of essential amino acids, fibres and antioxidants. It seems, in fact, that vitamin $\mathrm{C}$ supplementation can significantly reduce the incidence, duration and severity of a respiratory infection.

4. To reduce the environmental and psychological stress that can interact with a heavy training. 
5. To administer chemo - and immunotherapy: vaccinations against the endemic virus at the competition venue should be checked before the start. Athletes with a low titter of circulating immunoglobulins should receive prepared immunomodulators. According to Frolich, monthly injections of immunoglobulin would reduce the duration of infections in swimmers by nearly two bystanders compared to swimmers who received a placebo. In contrast, Lindberg and Berglund observed no clinical benefit induced by the administration of nasal IgA on world-class canoeists. The Merieux multitest, a cocktail of 7 common antigens, allows repeated evaluations of the immunocompetence during training: the antigens are inoculated in the intradermal centre with a standard applicator, and the sequential inspection of the skin allows the evaluation of the intensity of the local response immune. Salivary and mucosal immunoglobulin concentrations can also be monitored, although the link between low Ig levels and increased susceptibility to infection is weak. Since an immunosuppression mechanism could be represented by the accumulation of prostaglandins, the immune function can be assisted by the precautionary or therapeutic administration of NSAIDs such as indomethacin; however, opinions regarding this practice differ.

6. In the case of simple upper respiratory tract infection, it is generally advisable to reduce training, in association with local therapy, with a decongestant during the day and an antihistamine at night. However, if a viral infection becomes systemic, exercise should be done with great caution, as heavy training or competitions could increase the severity of the disease. The risk of viral myocarditis, a rare but well documented cause of sudden death during exercise, should not be overlooked. Signs of a systemic infection include fever, myalgia, fatigue, cough, vomiting, diarrhoea, and lymphadenopathy; Those who have these symptoms, especially if they are an amateur athlete, must refrain from physical activity and respect an adequate period of rest until the symptoms disappear.

\section{REFERENCES}

Corbi G, Polito R, Monaco ML, Cacciatore F, Scioli M, Ferrara F. et al. (2019). Adiponectin Expression and Genotypes in Italian People with Severe Obesity Undergone a Hypocaloric Diet and Physical Exercise Program". Nutrients, 11(9), 2195. https://doi.org/10.3390/nu11092195

Di Maio G, Monda V, Messina A, Villano I, Francavilla VC. (2020) Physical activity and modification of lifestyle induce benefits on the health status. Acta Medica Mediterranea., 36(3):1913-1919.

Francavilla VC, Bongiovanni T, Genovesi F, Minafra P, Francavilla G. (2015). Localized bioelectrical impedance analysis: how useful is it in the follow-up of muscle injury? A case report. Med Sport., 68:323-34.

Francavilla VC, Bongiovanni T, Todaro L, Di Pietro V, Francavilla G. (2017). Probiotic supplements and athletic performance: a review of the literature. Med. Sport., Vol. 70:247-59. https://doi.org/10.23736/S0025-7826.17.03037-X

Francavilla C, Bongiovanni T, Todaro L, Genovesi F, Francavilla G. (2016) Risk factors, screening tests and prevention strategies of muscle injuries in elite soccer players: a critical review of the literature. Med Sport., 69: 134-50.

Francavilla G, Francavilla C. (2013). Physical exercise is terapy - L'attività motoria è terapia. Medicina dello Sport - Minerva Medica., 66:625-8.

Francavilla C, Francavilla A, Braschi M, et al. (2007). Physical exercise and sport activities in patients with and without coronary heart disease. Monadi Arch Chest Dis., 87-95.

Francavilla C, Vitale F, Ciaccio Marcello et al. (2018). Use of saliva in alternative to serum sampling to monitor biomarkers modification in professional soccer players. Frontiers in Phsiology. https://doi.org/10.3389/fphys.2018.01828 
Gleesons M, Mcdonald Wa, Pyne Db, Clancy Rl, Cripps Aw, Francis Jl, Fricker Pa. (2000). Immune status and respiratory illness for elite swimmers during to 12-week trainings cycle. Int J Sports Med., 21: 302-307. https://doi.org/10.1055/s-2000-313

Mazzeo F, Motti ML, Messina G, et al. (2013). Use of nutritional supplements among south Italian students of Physical Training and Sport University. Current Topics in Toxicology., 9:21-26.

Messina A, Bitetti I, Precenzano F, lacono D, Messina G, Roccella M, Parisi. et al. (2018). Non-Rapid Eye Movement Sleep Parasomnias and Migraine: A Role of Orexinergic Projections. Front Neurol. 28:9:95. https://doi.org/10.3389/fneur.2018.00095

Messina A, De Fusco C, Monda V, Esposito M, Moscatelli F, Valenzano A, et al. (2016). Role of the orexin system on the hypothalamus-pituitary-thyroid axis. Front. Neural Circuits. 10. https://doi.org/10.3389/fncir.2016.00066

Monda V, Villano I, Messina A, Valenzano A, Salerno M, Signorelli S, et al. (2019). Aerobic exercise and orexin A: Role of sympathetic activity and redox system. J Biol Reg and Hom Ag., 33, 587-592.

Moscatelli F, Messina G, Valenzano A, Monda M, et al. (2015). Relationship between RPE and Blood Lactate after Fatiguing Handgrip Exercise in Taekwondo and Sedentary Subjects. Biol Med.

Moscatelli F, Messina G, Valenzano A, Monda V, Viggiano A, Messina A, Petito A, Triggiani Al, Ciliberti MA, Monda M, Capranica L, Cibelli G. (2016). Differences in corticospinal system activity and reaction response between karate athletes and non-athletes. Neurological Sciences., 37(12). https://doi.org/10.1007/s10072-016-2693-8

Niemen DC, Henson Da, Gusewitch G, Warren BJ, Dotson RC, Butterworth DE, Nehlsen-Cannarella SL. Physical activity immune and function in elderly women. Med Ski Sports Exerc 1993; 25: 823-831. https://doi.org/10.1249/00005768-199307000-00011

Patti A, Bianco A, Karsten B, Montalto MA, Battaglia G, et al. (2017). The effects of physical training without equipment on pain perception and balance in the elderly: A randomized controlled trial. Work. 57:23-30. https://doi.org/10.3233/WOR-172539

Pedersens BK, Nielsen HB. (1997). Acute exercise and immune the system. In: Pedersen BK (and). Exercise Immunology. Heidelberg, Germany Springer., 2: 5-38.

Peterses EM. (1997). Exercise, immunology and upper respiratory tract infections. Int J Sports Med., 18: S69-S77. https://doi.org/10.1055/s-2007-972702

Polito R, Francavilla VC, Ambrosi A, Tartaglia N, et al. (2020). The Orexin-A serum levels are strongly modulated by physical activity intervention in diabetes mellitus patients. Journal of Human Sport and Exercise, 15(2proc): S244-S251. https://doi.org/10.14198/ihse.2020.15.Proc2.15

Polito R, Monda V, Nigro E, Messina A, Di Maio G, Giuliano MT, et al. (2020). The important role of adiponectin and orexin-A, two key proteins improving healthy status: focus on physical activity. Front. Physiol., 11:356. https://doi.org/10.3389/fphys.2020.00356

Polito, R., Scarinci, A., Ambrosi, A., Tartaglia, N., Tafuri, D., Monda, M., Messina, A., Cimmino, F., Catapano, A., Sessa, F., Di Maio, G., Francavilla, V.C, Messina, G., \& Monda, V. (2020). The beneficial effects of physical activity and weight loss on human colorectal carcinoma cell lines. Journal of Human Sport and Exercise, 15(2proc), S252-S260. https://doi.org/10.14198/ihse.2020.15.Proc2.16

Valenzano A, Polito R, Trimigno V, Di Palma A, Moscatelli F, Corso G, Sessa F, Salerno M, Montana A, Di Nunno N, Astuto M, Daniele A, Carotenuto M, Messina G, Cibelli G, Monda V. (2019). Effects of Very Low Calorie Ketogenic Diet on the Orexinergic System, Visceral Adipose Tissue, and ROS Production. Antioxidants., 8(12). https://doi.org/10.3390/antiox8120643

Vincenzo Cristian Francavilla, Francesco Vitale, Marcello Ciaccio, Tindaro Bongiovanni, Claudia Marotta, Rosalia Caldarella, Lorenzo Todaro, Maurizio Zarcone, Roberto Muratore, Chiara Bellia, Giuseppe Francavilla And Walter Mazzucco. (2018). Use of Saliva in alternative to serum sampling to monitor 
biomarkers modifications in professional soccer players Frontiers in Physiology. https://doi.org/10.3389/fphys.2018.01828

Vincenzo Cristian Francavilla, Tindaro Bongiovanni, Lorenzo Todaro, Federico Genovesi, Giuseppe Francavilla. (2016). Risk factors, screening tests and prevention strategies of muscle injuries in èlite soccer players: a critical review of the literature Med. Sport., 69:134-50.

\section{(c) $(\mathrm{B} \$(\mathrm{EY}$}

This work is licensed under a Attribution-NonCommercial-NoDerivatives 4.0 International (CC BY-NC-ND 4.0). 